\section{In tern ationa l \\ Journal of}

Medical

and Surgical Sciences

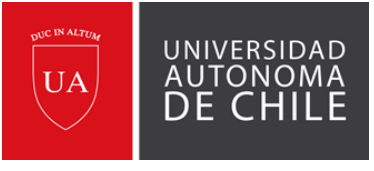

\title{
Bilateral canine space infection secondary to odontogenic origin. A rare case report.
}

\author{
Mayur Janardan Gawande, ${ }^{1}$ Mithilesh Kumar Gupta ${ }^{1}$ \& Manu Goel. ${ }^{1}$
}

\section{ABSTRACT}

Odontogenic infections $(\mathrm{OI})$ are a major reason for consultation in dental practice. They affect people of all ages, and most of them respond well to current medical and surgical treatments. The aim of this paper is to emphasize different and rare etiological factors that can play a role in odontogenic abscesses, which can lead to complications. A 54-year-old man complaints of pain and swelling on the upper front-side of his face. Physical examination of the face showed enlargement and swelling in the upper front region. Panoramic radiograph images showed a radiolucent appearance of the canine region. Based on the results of the clinical and radiographic examinations, the patient was diagnosed with a bilateral canine space infection. The treatment performed was FNAC (Fine neddle aspiration cytology) from canine region followed by intraoral drainage incision at the most prominent part of swelling (Modified Hiltons method), $5 \mathrm{ml}$ of pus was removed, postoperative dressing was applied and regular follow-up was done with continued antibiotic therapy. Bilateral canine space abscess is a rare odontogenic infection that can cause life-threatening complications. The success of treatment requires early recognition, determination of etiological factors, and appropriate medical and surgical management. To perform treatment of odontogenic infections, the Oral \& Maxillofacial Surgeon should understand the terminology regarding infectious diseases and pathophysiology. The success of treatment requires early recognition, determination of etiological factors, and appropriate medical and surgical management.

Keywords: Odontogenic Infections, Bilateral canine space infection.

\section{INTRODUCTION}

The canine space (also named infra-orbital space), is a fascial space of the head and neck (sometimes also named fascial spaces or tissue spaces). It is a thin potential space on the face, and is paired on either side. It is located between the levator anguli oris muscle inferiorly and the levator labii superioris muscle superiorly (Tamales et al., 2016; Petersen, 1996). The term is derived from the fact that the space is in the region of the canine fossa, and that infections originating from the maxillary canine tooth may spread to involve the space. Infra-orbital is derived from infra- meaning below and orbit which refers to the eye socket. The oral cavity is a breeding ground for various microorganisms, where an injury can occur inevitably (Tamales et al., 2016). Microorganisms normally present in the oral cavity may cause infection if; firstly, the nature of the microorganism is altered both in quality and quantity; secondly, bacteria penetrate the oral mucosa and tooth pulp; and thirdly, the immune system and cellular defenses are disrupted. Any combination of the above-mentioned incidents can result in acute or chronic infections. The acute condition is usually accompanied by severe swelling and pain with systemic manifestations of prolonged malaise and fever, while the chronic forms can develop from partial healing of acute circumstances, weak attacks or strong defenses (Petersen, 1996). Infection is a process that involves the proliferation of microorganisms that cause the body's defense reaction, a process called inflammation. Inflammation is a vascular reaction, which results in the delivery of fluids, dissolved substances and blood cells from the blood to the interstitial tissues of the injured or necrotic region. Acute inflammation 
is an immediate reaction to injury or cell death. The main signs of inflammation are dolor (pain), rubor (redness), calor (heat), tumor (swelling) and functio laesa (alteration of function). To understand the difference between the types of inflammation, it is considered important to know and determine treatment therapy (Fragiskos, 1956). Odontogenic infections are the most commonly encountered infections and a worldwide problem and, hence, the main reason for seeking proper dental care (Lopez-piriz et al., 2007).

Odontogenic infections can develop from damaged teeth, trauma to the root area of teeth, pathogens in the mouth or the presence of inflammatory mediators that rapidly infiltrate nearby areas, such as submandibular trigonum or canine fossa. This can lead to the formation of fluids in soft tissues and abscess formation. In the case of a canine fossa abscess, formation of fluids can extend into the cranial region. Airway obstruction, periorbital abscess, and intracranial abscess are the most common and familiar symptoms when infection is increased (Agacayak et al., 2013). Odontogenic infections are common and can become a dangerous health problem when life-threatening complications occur. These infections can spread through the bone and periosteum against nearby structures increasing the risk of septicemia and death for affected patients. Canine space abscess is an odontogenic infection that can cause these life-threatening complications. The success of treatment requires early recognition, determination of etiological factors, and appropriate medical and surgical management (Agacayak et al., 2013; Veronez et al., 2014). To perform treatment of odontogenic infections, the dentist should understand the terminology regarding infectious diseases and pathophysiology.

Odontogenic infections stem from a variety of microbes, such as aerobic bacteria and facultative anaerobes. Anatomical factors play a key role in the presentation of bacterial infections that spread beyond the confines of the jaw. The spread of infection tends to follow the most opposite line determined by the bone and periosteum, muscle and fascia. Determining the severity of infection, evaluating the host defense, surgical management, medical support, administering antibiotics and periodic evaluation of patients are the main routes of odontogenic infection management. Three main factors to consider when determining the severity of infection in the head and neck are location anatomy, rate of progression, and airway compromise (Ferrera et al., 1996). The spread of infection to the canine fossa usually originates from the maxillary canine or the upper premolar teeth, often seen above the buccinator muscle. This swelling removes the nasolabial folds, the space near the lower eyelid, and, therefore, early management is very important to avoid circular orbital infections. There is a risk of spread to the cranium through the external angle of the vein that can lead to thrombosis (Ferrera et al., 1996).

\section{CASE REPORT}

A 54-year-old man arrived at the Department of Oral \& Maxillofacial Surgery at Swargiya Dadasaheb Kalmegh Smruti Dental College \& Hospital, Nagpur, with complaints of pain and swelling on the upper front-side of his face (Figure 1). The patient had a history of pain and fever. Physical examination of the face showed enlargement and swelling in the upper front region of the jaw (Figure 2). Panoramic radiograph images showed a radiolucent appearance of the canine region (Figure 3). Based on the results of the clinical and radiographic examinations, the patient was diagnosed with a bilateral canine space infection. The treatment performed was FNAC (Fine neddle aspiration cytology) (Figure 4) from canine region followed by intraoral drainage incision at the most prominent part of swelling (Modified Hiltons method) (Figure 5), approximetely $5 \mathrm{ml}$ of pus was removed and postoperative dressing was applied (Figure 6). Further endodontic treatment was performed with $11 \& 21$, followed by continued antibiotic therapy.

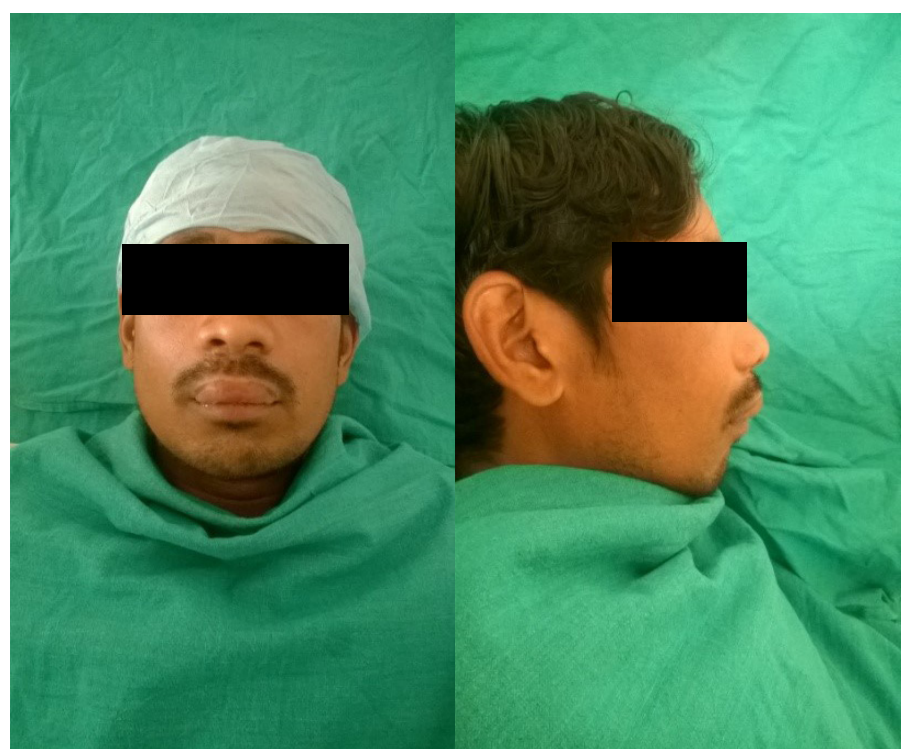

Figure 1. Extraoral swelling over the upper lip and oblitering the nasolabial fold.

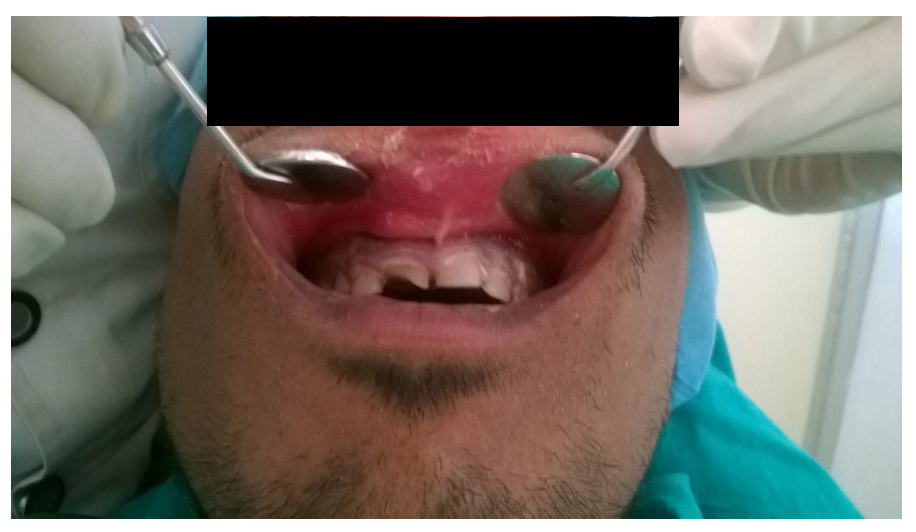

Figure 2. Intraoral swelling from canine to canine region. 


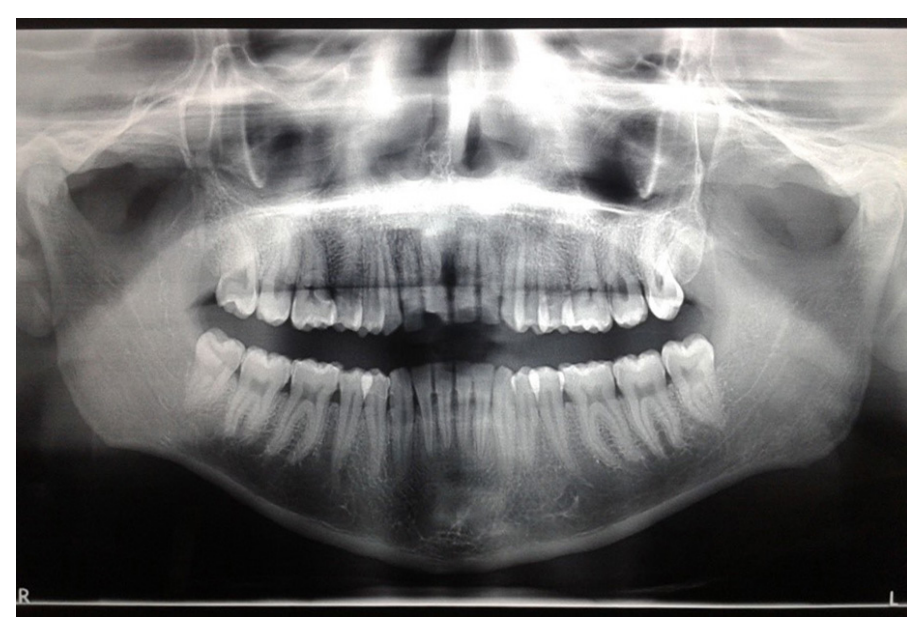

Figure 3. Panaromic radiograph showing radiolucency at Canine region.

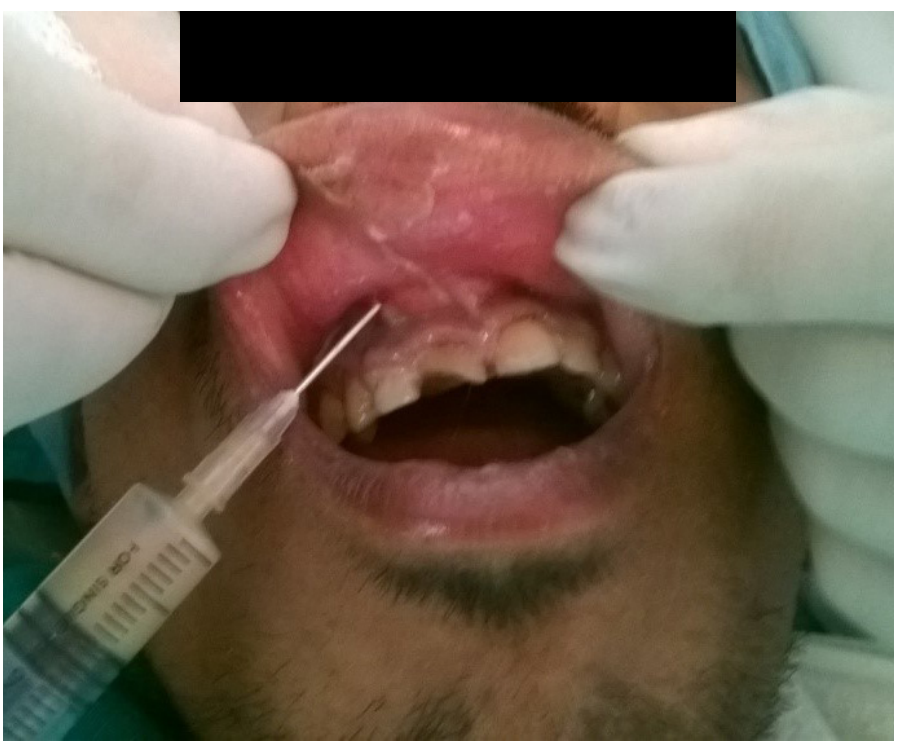

Figure 4. Fine neddle aspiration from canine region.

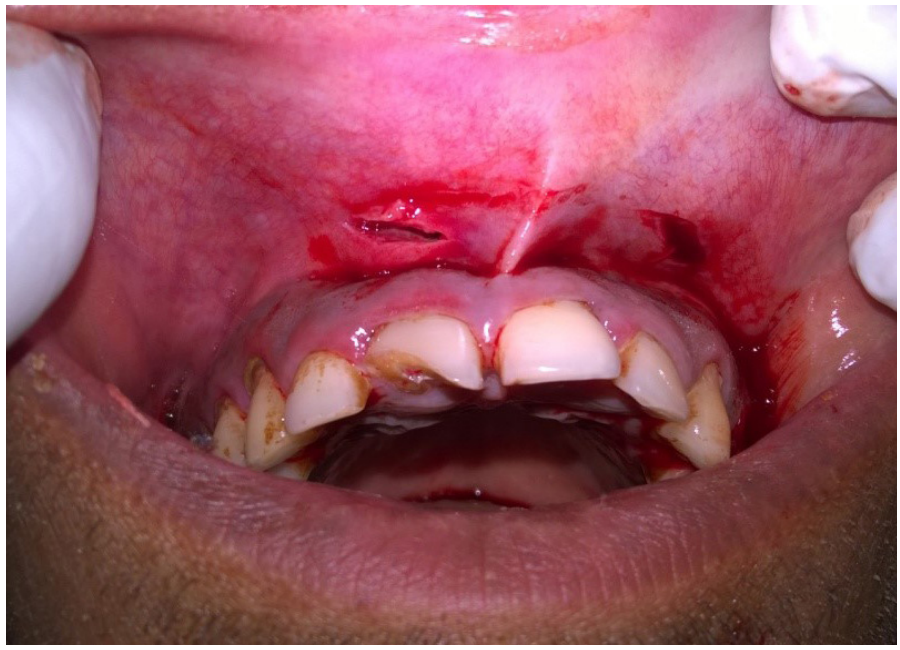

Figure 5. Stab Incision by modified Hiltons method on both the side.

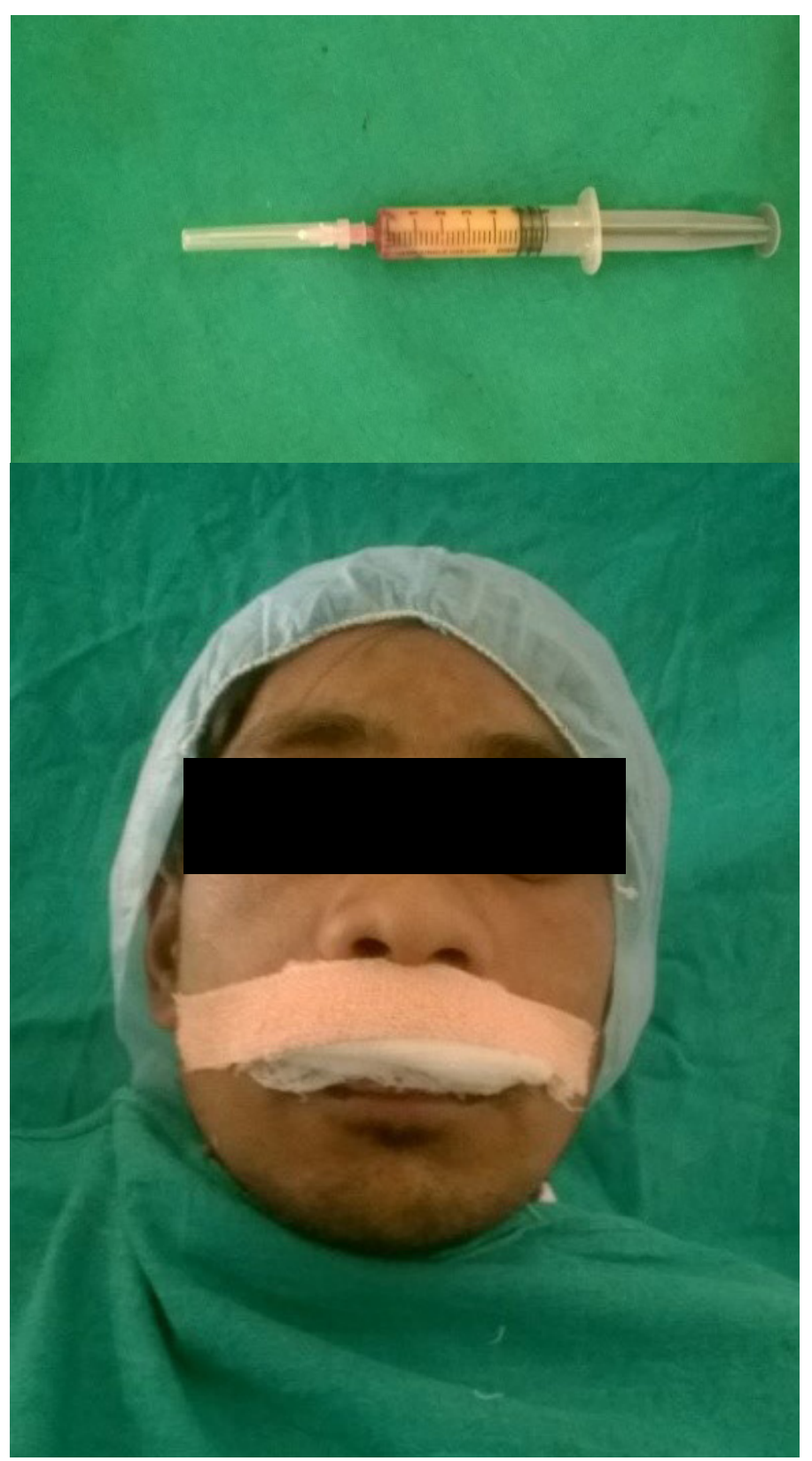

Figure 6. Postoperative dressing applied.

\section{DISCUSSION}

The underlying infectious process may be of odontogenic or non-odontogenic origin. Odontogenic infection (Ol) originates in teeth or in surrounding tissues, affecting the periapical bone from where it spreads towards either neighboring structures (continuous propagation) or structures located further away (distant propagation) (Velasco \& Soto, 2012). Odontogenic infections are the most frequent presentations, $70-90 \%$ originating from pulp necrosis, periodontal disease, pericoronitis, granulomas, apical cysts or complications of dental procedures. Non-odontogenic infections, in turn, are associated to maxillofacial fractures, submandibular sialoadenitis, infections of the salivary glands, tumor or cystic lesions, and infections of pharyngeal or tonsillar origin, among others (Flynn et al., 2006, Lugo et al., 2014). 
The literature describes odontogenic infections as the most common cause of head and neck ailments. Umeda et al. (2003) presented 9 cases and reviewed the English language literature, documenting 125 infections of odontogenic origin. They reported periapical infections of the second and third mandibular molars as being the most frequent origin $(70-80 \%)$, due to the fact that the roots of these teeth typically extend beneath the mylohyoid muscle, producing infection that spreads into the submaxillary space, and from there to the sublingual and submental spaces, consecutively. Flynn et al. (2006) published a study of 49 cases of severe odontogenic infections with involvement of the deep lying spaces. Of these cases, $68 \%$ were associated to inferior third molars, $22 \%$ to pericoronitis, and the rest to other mandibular posterior teeth. Our case is consistent with the origin described in the literature, involving a semi-impacted third mandibular molar with pericoronitis that evolved into a phlegmon in the floor of the mouth. Kurien et al. (1997) carried out a comparative study of the causes of Ludwig's angina in children and adults. They identified a dental origin in $52 \%$ of the adults, and $39 \%$ suffered predisposing systemic diseases, such as poorly managed diabetes, alcohol abuse or immunosuppression.

Empirically, amoxicillin antibiotic therapy with potassium clavulanate and metronidazole, together with drainage incisions, provides excellent treatment outcomes for all patients. Beta-lactam antibiotics have been used to treat odontogenic infections because they are very effective against certain bacteria, cost effective, and have little side effects. Increased beta-lactam resistance, due to the emergence of organisms that produce beta-lactamase, has caused concern about the effectiveness of beta-lactam antibiotics in recent years (Mathew et al., 2012).

Canine space infections may occur by spread of infection from the buccal space. Signs and symptoms of a canine space abscess might include swelling that obliterates the nasolabial fold. If left untreated, infections of this space will eventually spontaneously drain via the medial or lateral canthus of the eye, as this is the path of least resistance. Treatment is usually by surgical incision and drainage, and the incision is placed inside the mouth to avoid a facial scar. Rarely, when infections of the canine space erode into the infra-orbital vein or the inferior ophthalmic vein (via the sinuses), there can be spread via the common ophthalmic vein through the superior orbital fissure and into the cavernous sinus. This can result in septic cavernous sinus thrombosis, which is a rare, but life-threatening condition (Hupp et al., 2008).

\section{CONCLUSSION}

Odontogenic infections can be life-threatening with the formation of abscesses around the tissue structure. It is therefore important that dentists are aware of possible ways in which odontogenic infections can spread and know how to deal with such complications in case of an emergency. Bilateral canine space infection is an odontogenic infection that can cause life-threatening complications. The success of treatment requires early recognition, determination of etiological factors, and appropriate medical and surgical management.

\section{REFERENCES}

Agacayak S, Atilgan S, Belgin G. Case report: canine fossa abscess; a rare etiological factor: the lower canine tooth. J Int Dent Med Res. 2013;6: 36-39.

Ferrera PC, Busino ப, Snyder HS. Uncommon complications of odontogenic infections. Am J Emerg Med. 1996;14: 317-322.

Flynn TR, Shanti RM, Levi MH, Adamo AK, Kraut RA, Trieger N. Severe odontogenic infections, part 1: prospective report. J Oral Maxillofac Surg. 2006;64: 1093-103.

Fragiskos FD. The role of oral surgery in orthodontic treatment planning. Am J Orthodontic Dentofac Ortophedic. 1956;42: 279-287.

Hupp JR, Ellis E, Tucker MR. Contemporary oral and maxillofacial surgery (5th ed.). St. Louis, Mo.: Mosby Elsevier; 2008. 317-333.

Kurien M, Mathew J, Job A, Zacharia N. Ludwig's angina. Clin Otolaryngol Allied Sci. 1997;22: 263-5.

Lopez-piriz L, Aguilar L, Gimenez JM. Management of odontogenic infection of pulpa and periodontal origin. Med Oral Patol Oral Cir Bucal 2007;12: 154-159.

Lugo AFG, Ravago MGC, Martinez RAG, Peltrini RJZ. Ludwig's angina: A report of two cases. Rev Esp Cir Oral Maxilofac. 2014;36: 177-81.

Mathew GC, Ranganathan LK, Gandhi S. Odontogenic maxillofacial space infections at a tertiary care center in North India: a five-year retrospective study. Int J Infec Diseases 2012;16: 296-302.

Petersen GW. Oral surgery 1 ed. Philadelphia: WB Saunders Company; 1996. 191-197.

Tamales D, Dewi N, Rosida L. Extract of haruan (channa striata) extract increasing reepithelialisation count in wound healing process on wistar rat's buccal mucosa. J Dentomaxillofac Sci. 2016;1(1): 12-15.

Umeda M, Minamikawa T, Komatsubara H, Shibuya Y, Yokoo S, Komori T. Necrotizing fasciitis caused by dental infection: a retrospective analysis of 9 cases and a review of the literature. Oral Surg Oral Med Oral Pathol Oral Radiol Endod. 2003;95(3): 283-90.

Velasco I, Soto R. Principles for the treatment of odontogenic infections with different levels of complexity. Rev Chil Cir. 2012;64: 586-98.

Veronez B, De-Matos, Monnazzi MS. Maxillofacial infection. A retrospective evaluation of eight years. Braz J Oral Sci. 2014;13: 98-103. 